\title{
Creating a reference guide to improve management of inpatients with influenza and other respiratory viral infections
}

\author{
Author: Rishi Raj Banerjee ${ }^{\mathrm{A}}$
}

\section{Introduction}

Seasonal respiratory viruses are a significant cause of morbidity and mortality, and management of inpatients with influenza and other respiratory viruses has important implications for clinical outcomes and patient flow. There is currently no easy-to-access reference guide at Lewisham and Greenwich NHS Trust to guide clinicians on post-exposure prophylaxis, treatment indications or appropriate isolation duration for these patients. Microguide, an application freely available on mobile phones, is a well-established multi-trust platform that guides antibiotic use but has not traditionally been used for respiratory virus management. This quality improvement (QI) project looks to improve clinicians' knowledge base and confidence in managing patients by creating a respiratory virus reference section on Microguide.

\section{Methods}

The initial phase used a 10-question survey, aimed at junior doctors in the acute medical team, to gauge current knowledge of management, awareness of currently available resources and enthusiasm for a new reference guide. A clinical reference section was then created on Microguide, using existing trust guidance and Public Health England guidelines as primary resources. The next stage, which is currently in progress, is to update the trust Microguide application so it reflects the new update, and then raise awareness with clinicians. Finally, the survey will be re-performed to see if knowledge-base and confidence in managing patients have improved.

\section{Results}

The initial survey was completed by 20 junior doctors at Queen Elizabeth Hospital (Woolwich), who ranged in grade from foundation year 1 to specialty trainee. Of the first eight multiple choice questions pertaining to clinical management of inpatients, six questions were answered incorrectly by over $50 \%$ of those surveyed. Question 9, which assessed awareness of current trust guidance, demonstrated that over $70 \%$ did not know where to access guidelines. One hundred per cent of those surveyed felt a new reference guide would be helpful to their clinical practice.
The next stage (in progress) is to roll out the updated Microguide application, raise awareness and re-perform the survey.

\section{Discussion}

The initial survey suggests lack of knowledge of management of inpatients with influenza and other respiratory virus infections among Lewisham and Greenwich clinicians, unawareness of how to access relevant trust guidance and an enthusiasm for a new reference guide.

I hope to roll out the Microguide update and re-perform the survey by February 2020. Once that cycle is complete, there will be some indication as to whether an updated and expanded Microguide platform is a useful accessory for clinicians in the management of inpatient respiratory virus infections.

\section{Conflicts of interest}

None declared. 